\title{
Apoyo metodológico musical de las lecciones de inglés como tecnología pedagógica
}

\section{Musical methodological support of English lessons as pedagogical technology}

Alex Geovanny Inca Chunata. ${ }^{1}$ Esthela Isabel Colcha Guashpa. ${ }^{2}$ Sandra Leticia Guijarro Paguay. ${ }^{3} \&$ Leonardo Mauricio Martínez Paredes. ${ }^{4}$

\begin{abstract}
.
This article describes the feasibility and effectiveness of music support in the English language teaching process by introducing new technologies. Based on 10 years of personal experience teaching English with songs that create a means of immersion in the atmosphere of the language. The role of songs (as one of the types of oral communication) significantly improves the assimilation and expansion of students' vocabulary, in the development of the sense of language and the knowledge of its stylistic characteristics. For the study, a group of 70 pre grade students was consider. This pedagogical technology helps to learn the English language and develops an interest in the culture of students who expand their business frontiers in international trade. It also develops students' sensitivity in pronunciation, rhythm and emotionality of the English language, helps to recognize English by ear, expands vocabulary, motivates language learning and develops aesthetic taste.
\end{abstract}

Keywords: English, Pedagogy, Music, Rhythm.

\section{Resumen:}

\footnotetext{
${ }^{1}$ Colegio de Artes Conservatorio Nacional de Música Quito, Ecuador, email alex.inca@yahoo.es

${ }^{2}$ Escuela Superior Politécnica de Chimborazo, Facultad de Ciencias Pecuarias. Riobamba, Ecuador. email e.colcha@espoch.edu.ec

${ }^{3}$ Escuela Superior Politécnica de Chimborazo, Facultad de Mecánica. Riobamba, Ecuador. email sandra.guijarro@espoch.edu.ec

${ }^{4}$ Escuela Superior Politécnica de Chimborazo, Facultad de Mecánica. Riobamba, Ecuador. email lmartinez@espoch.edu.ec
} 
Este artículo describe la viabilidad y eficacia del apoyo musical en el proceso de enseñanza del idioma inglés introduciendo nuevas tecnologías Basado en la experiencia personal de 10 años enseñando inglés con canciones que crean un medio de inmersión en la atmósfera del idioma. El papel de las canciones (como uno de los tipos de comunicación oral) mejora de manera significativa la asimilación y expansión del vocabulario de los estudiantes, en el desarrollo del sentido del lenguaje y el conocimiento de sus características estilísticas. Para el estudio se ha considerado un grupo de 70 estudiantes de pre grado Esta tecnología pedagógica ayuda a aprender el idioma inglés y desarrolla un interés en la cultura de los estudiantes que amplían sus fronteras de negocios en el comercio internacional. También desarrolla la sensibilidad de los estudiantes en la pronunciación, el ritmo y la emotividad del idioma inglés, ayuda a reconocer el inglés de oído, amplía el vocabulario, motiva a aprender el idioma y desarrolla el gusto estético.

Palabras clave: Enseñanza, inglés, Pedagogía, Música, Ritmo.

\section{Introducción.}

La consideración de este tema está determinada por la necesidad de crear y mejorar constantemente nuevas tecnologías pedagógicas para la enseñanza del idioma inglés, con estudiantes de pre grado, asegurando la efectividad de su estudio. Esta tarea adquiere especial importancia en el contexto de la intensificación del proceso educativo.

De manera experimental se quiere corroborar la idoneidad y efectividad de la experiencia de enseñar el idioma inglés usando el acompañamiento musical y metodológico durante las clases grupales. La descripción teórica es insuficiente y requiere de técnicas que permitan asimilar el conocimiento de manera natural como lo exponen algunos autores de artículos que cubren ciertos aspectos del estudio del problema: el papel y la importancia de la música en el proceso educativo.

El investigador (Toscano Fuentes, 2012) comparte su experiencia de usar música y material de canciones en el proceso de enseñar a los estudiantes de idioma inglés y concluye que, además del desarrollo y la formación de habilidades del habla, también existe una "asimilación integral por parte de los estudiantes de información de naturaleza geográfica, lingüística, regional y cultural y estética regional", que contribuye a la familiarización de los estudiantes con patrimonio espiritual y cultural del país de la lengua estudiada (Dandrea, 2018).

La investigadora (Anaya-Durand, 2010) enfatiza que a pesar del hecho de que actualmente el material musical está presente en casi todos los cursos de capacitación en un idioma extranjero y el principal resultado del uso de la música en una clase de idioma extranjero es aliviar la ansiedad de los estudiantes y crear emociones positivas, lo que 
contribuye al desarrollo del interés en el tema que se estudia y, por lo tanto, mejora la calidad de la educación, el tema del uso de la música como base para la enseñanza de lenguas extranjeras no está ampliamente cubierto.

El investigador (Madariaga, 2011) considera el problema de la motivación y el papel de la música en la enseñanza de un idioma extranjero, enfatiza que el uso de ejercicios musicales en el aula "ayuda a armonizar, alinear al grupo y hacer que el aprendizaje sea más interesante, creativo, alegre y fructífero. Los estudiantes memorizan mejor las palabras nuevas y asimilan el material de aprendizaje, perciben más fácilmente el habla en un idioma extranjero de oído, cometen menos errores en la pronunciación. Cantar canciones es natural. Aprender un idioma extranjero con la ayuda de canciones es una forma natural y, por lo tanto, conveniente y exitosa de aprender una nueva cultura, otro mundo aún desconocido.

El investigador (Espinoza, 2015) considera el tema del uso de material de canciones auténticas en las clases de inglés como lengua extranjera. Se destaca el papel especial de la música y las canciones como medio para enseñar vocabulario, gramática y fonética; comunicación, estudios lingüísticos. Espinoza cree que para un maestro que se acerca de manera creativa a su clase, el material de canción auténtico siempre puede ser una buena ayuda para organizar el proceso educativo. Acerca de la idea de usar música al aprender inglés, porque es la música la que afecta la esfera emocional.

Para el investigador (Blasco, 2001) la música como un arte que tiene la mayor fuerza de impacto emocional, lo que ha hecho de la música un tema obligatorio del sistema educativo. Su trabajo presenta los resultados de un estudio experimental que demuestra claramente la posibilidad y la conveniencia de utilizar la composición para mejorar la motivación en el aprendizaje de idiomas extranjeros, revela los patrones del impacto del arte musical en los estudiantes, se intenta desarrollar un programa para optimizar el proceso educativo.

Los investigadores nacionales también contribuyeron al estudio de este tema. (Inca, 2020) publica una revisión de la literatura sobre el tema: "el uso de música y canciones en el estudio del inglés con estudiantes universitarios y muestra una estrecha relación entre la música y el lenguaje, basada en varios estudios en el campo de la andragogía, la sociolingüística, la psicolingüística y concluye que el uso de la música en el estudio del inglés ayuda a los estudiantes universitarios a adquirir conocimientos gramaticales fijos y también ayuda a expandir la comunicación intercultural.

Un estudio de estos y otros artículos científicos sobre este tema llevó a la conclusión de que no hay trabajos que examinen el papel del acompañamiento musical y metodológico de las clases de canciones como tecnología pedagógica en el estudio del idioma inglés. 
Una importante tarea pedagógica de enseñar el idioma inglés se actualiza en estrecha conexión con la solución de una tarea educativa importante: la formación de cualidades morales y el desarrollo multifacético de la personalidad de cada estudiante. La tecnología pedagógica propuesta cumple plenamente la tarea de educar a los estudiantes.

Este trabajo destaca el importante papel que juega el acompañamiento musical y metodológico en el proceso de enseñanza de una lengua extranjera, en particular el inglés, que es la lengua materna de los artistas y grupos de fama mundial como: Bob Dylan, Queen, The Beatles, Madona, Robbie Williams, U2, Coldplay y muchos otros, que son de gran agrado en la etapa juvenil de los estudiantes de pre grado. Las obras maestras musicales de estos y otros artistas motivan a quienes desean aprender inglés. La experiencia del autor del artículo muestra que es extremadamente difícil o incluso casi imposible lograr altos resultados en los estudiantes que estudian inglés como segunda lengua extranjera (desde el nivel cero). Los diferentes niveles de habilidades y receptividad de los estudiantes a un nuevo idioma dificultan el desarrollo rápido y exitoso de un nuevo idioma.

\section{Metodología.}

La investigación de tipo experimental se ejecutó durante las clases prácticas grupales del idioma inglés en la Escuela Superior Politécnica de Chimborazo, así como durante la participación de uno de los autores en el Instituto de Música de Quito. Se realizó el experimento durante 2 años en 8 grupos de estudiantes (15-20 estudiantes por grupo): 2 grupos de estudiantes de 1er nivel; 2 grupos de estudiantes de 2 nivel; 2 grupos de estudiantes de 3 nivel; 2 grupos - 4 nivel.

A los estudiantes se les dio la tarea de dominar el nuevo vocabulario, la pronunciación fonética correcta de las nuevas palabras. La tarea del profesor es usar el acompañamiento metodológico musical con la inclusión de la interpretación de la canción para que esta etapa del trabajo en el aula brinde a los estudiantes alivio psicológico después de una encuesta estresante o trabajo en nuevo material.

La tarea del profesor es seleccionar esas canciones para el acompañamiento musical y metodológico de las clases, de modo que sean diferentes emocionalmente, porque las canciones siempre están llenas de la energía de las personas que hablan este idioma. Cada idioma tiene su propio ritmo, sus propios tonos de sonidos; A través de los ritmos musicales se revela la belleza del lenguaje, su lado emocional. Para aprender un idioma extranjero, es importante escuchar cómo otras personas expresan diferentes emociones en su música.

La composición de canciones en inglés como acompañamiento musical y metodológico es utilizada activamente por los autores de este artículo en la práctica en el proceso de enseñar a los estudiantes el idioma inglés. Utilizando esta tecnología pedagógica, desde 
la etapa de estudio del alfabeto hasta el lenguaje más complejo, los tiempos gramaticales y los modos subjuntivos (Modo Subjuntivo). Las letras recomendadas para los estudiantes y su rendimiento de sonido están disponibles para los estudiantes a través de recursos de Internet, y también son proporcionadas por el maestro en forma de transmisión de archivos de sonido en medios.

\section{Resultados.}

El experimento mostró cómo mejoraron los resultados de aprendizaje de los estudiantes en grupos de estudiantes de 17-23 años (70 estudiantes), en el aula donde se utilizó tecnología pedagógica como el acompañamiento musical y metodológico de las clases en inglés. Se evalúan aspectos: mejorar la pronunciación, memorizar vocabulario nuevo, aumentar el interés de los estudiantes.

La Figura 1 indica que los estudiantes de 4 grupos de nivel 1 y 2 tuvieron dificultades significativas para dominar la pronunciación y el nuevo vocabulario en inglés. Solo el $10 \%$ tenía alto el resultado de memorizar vocabulario, mejorar la pronunciación, confianza en la pronunciación correcta y en su conocimiento; el 30\% - obtuvo un resultado medio y $60 \%$ - obtuvo un bajo nivel de rendimiento.

Figura 1. Comparativo de niveles de aprendizaje obtenidos de manera tradicional



Fuente: Elaboración propia. 
Figura 2. Resultados en los niveles de aprendizaje obtenidos posterior al método musical



Fuente: Elaboración propia.

La Figura 2 muestra que las clases con acompañamiento musical y metodológico de las clases en el idioma inglés, con la inclusión de la etapa de "canción" en las clases, dieron una efectividad significativa en el logro de los objetivos. La mayoría de los estudiantes tienen un éxito en la obtención de un nivel alto del dominio del idioma (64\%) y medio (25\%) en la pronunciación y el vocabulario de aprendizaje, y los resultados bajos (10\%) se minimizan. Los estudiantes tienen más confianza, demuestran interés no solo en las clases intensivas en un idioma extranjero, sino también en estudiar la cultura globalizada.

Un experimento con 4 grupos de tercer y cuarto nivel (80 estudiantes) mostró que antes de la inclusión del acompañamiento musical y metodológico en el curso de las clases de inglés, la interpretación, traducción, y léxico seguía siendo limitado, no había interés en la lengua estudiada. En la figura 3 se muestran los resultados de aprendizaje obtenidos con la aplicación del método tradicional en consecuencia: $65 \%$ obtuvo un resultado bajo, $25 \%$ obtuvo un resultado medio, $10 \%$ obtuvo un resultado alto. 
Figura 3. Resultados en los niveles de aprendizaje obtenidos por el método tradicional.

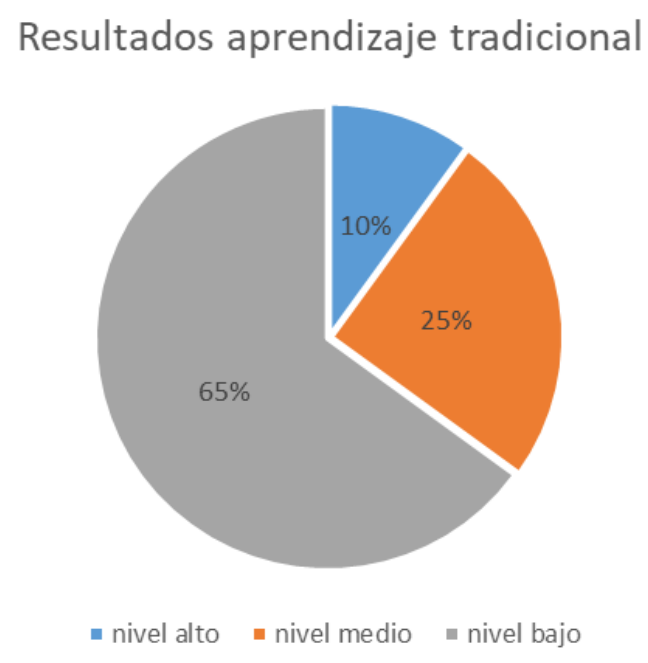

Fuente: Elaboración propia

Figura 4. Resultados en los niveles de aprendizaje obtenidos posterior al método musical

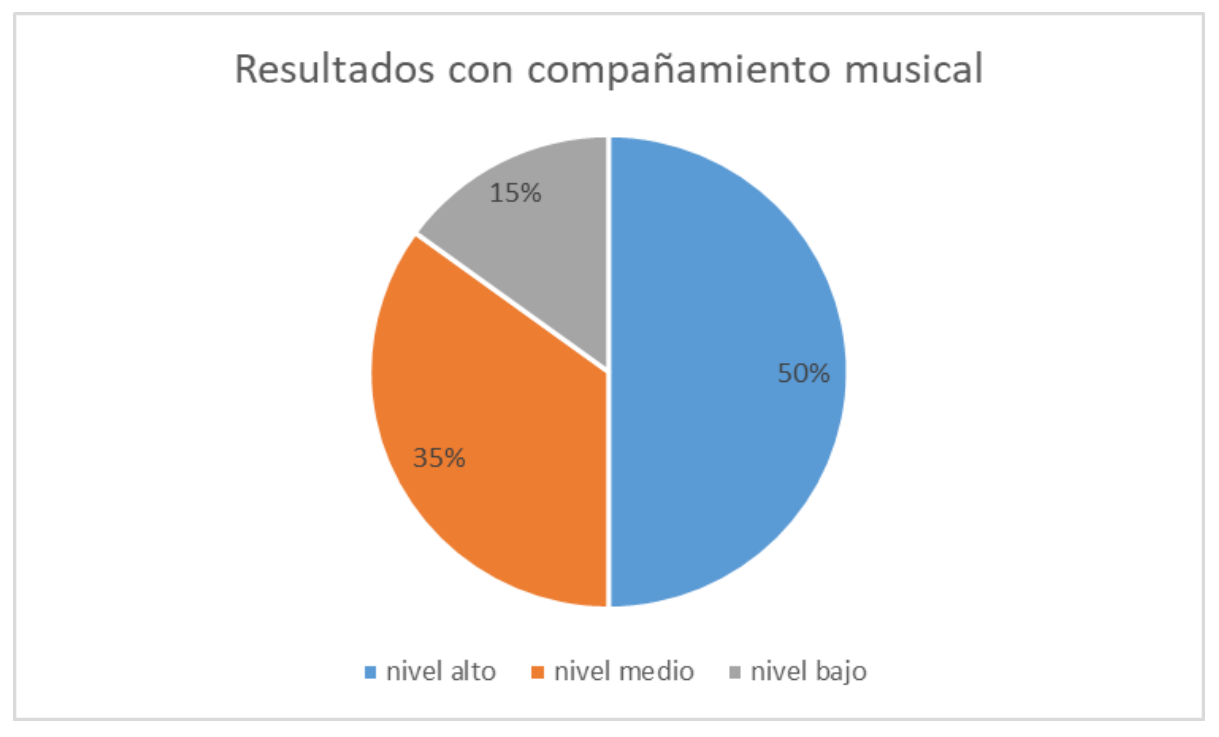

Fuente: Elaboración propia

La Figura 4 muestra los resultados de la enseñanza del idioma inglés en grupos de tercer y cuarto nivel en las clases de inglés midiendo los parámetros del vocabulario y pronunciación. Como se muestra el 50\% de los estudiantes logró un resultado alto y el $35 \%$ obtuvo un resultado medio, y apenas el $15 \%$ del resultado corresponde a un nivel bajo. 
Así, el experimento mostró que los estudiantes que estudiaban inglés con el uso de tecnología pedagógica como el apoyo musical y metodológico para las clases, dominaron con éxito el nivel de inglés requerido por el programa educativo. Al mismo tiempo, la asistencia a clases fue casi del 95\%, lo que indica el gran interés de los estudiantes en las clases. Los estudiantes, gracias al acompañamiento musical y metodológico, no solo dominaron con seguridad el material fonético y léxico en una forma ligera, sino que se familiarizaron con la cultura de la canción. En comparación con los grupos en los que se trabajó de acuerdo con la metodología clásica y tradicional de las clases.

Sobre el aspecto psicológico y pedagógico desarrollado mediante el acompañamiento metodológico musical del idioma inglés. Quizás los métodos más efectivos para enseñar un idioma puedan reconocerse como aquellos que convierten el aprendizaje en un juego, en un emocionante proceso creativo. Esto se relaciona directamente con el uso de la composición de canciones en la enseñanza de un idioma extranjero.

Está comprobado que las emociones positivas afectan significativamente el aprendizaje. La percepción emocional del material ayuda al alumno a concentrarse y aprender mejor el material estudiado. Por lo tanto, una de las tareas más importantes de un maestro de un idioma extranjero debe ser el deseo de hacer que el aprendizaje sea brillante y memorable, para que el aprendizaje del idioma sea agradable y placentero. La buena música contribuye a un trabajo más efectivo del maestro, ya que mejora el estado de ánimo y aumenta la motivación de los estudiantes.

Es tarea del maestro inculcar las habilidades de la cultura del habla y el uso de una canción en el idioma estudiado le permite dominar rápida y exitosamente el vocabulario temático, que generalmente requiere un tiempo de entrenamiento especial. Las canciones de cierto contenido temático le permiten ahorrar tiempo de entrenamiento. Entonces, por ejemplo, para estudiar efectivamente las características de las vocales del alfabeto en inglés, la canción "Imagine_John Lennon" o una canción fácil y provocativa con palabras que a los estudiantes realmente les gustan y que rápidamente son recordadas por ellos: "Otherside_Red Hot Chilli Peppers",

En la etapa de estudio de los temas para enriquecer el vocabulario y la interpretación propia de cada individuo "Estranged_Guns and roses", "The Piano Man_Billy Joel”, son relevantes las canciones que le permiten recordar rápidamente el vocabulario temático y conducen a que el estudiante elabore su propia traducción e interperetación con un estilo juvenil.

"Have you ever seen the rain_Credence" es una de las favoritas que incluso permiten a los estudiantes mejorar su estado de ánimo al momento de aprender. La letra de la canción se muestra en la tabla 1 y su respectiva traducción 
Tabla 1. Canción utilizada para vocabulario y pronunciación en inglés

Alguna vez has visto la lluvia?

Alguien me dijo hace mucho tiempo

Hay calma antes de la tormenta

Lo sé, ha estado viniendo por algún tiempo

Cuando termina, entonces dicen

Lloverá un día soleado

Lo sé, brillando como agua

Quiero saber, ¿alguna vez has visto la lluvia?

Quiero saber, ¿alguna vez has visto la lluvia?

¿Vienes en un día soleado?

Ayer y días antes

El sol es frío y la lluvia es dura

Lo sé, ha sido así todo el tiempo

Hasta siempre para siempre

A través del círculo, rápido y lento

Lo sé, no puede parar, me pregunto

Quiero saber, ¿alguna vez has visto la

lluvia?

Quiero saber, ¿alguna vez has visto la lluvia?

¿Vienes en un día soleado?

$\mathrm{Si}$

Quiero saber, ¿alguna vez has visto la lluvia?

Quiero saber, ¿alguna vez has visto la lluvia?

¿Vienes en un día soleado?

\section{Have You Ever Seen the Rain?}

Someone told me long ago

There's a calm before the storm

I know, it's been comin' for some time

When it's over, so they say

It'll rain a sunny day

I know, shinin' down like water

I want to know, have you ever seen the rain?

I want to know, have you ever seen the rain

Comin' down on a sunny day?

Yesterday and days before

Sun is cold and rain is hard

I know, been that way for all my time

'Til forever on it goes

Through the circle, fast and slow

I know, it can't stop, I wonder

I want to know, have you ever seen the rain?

I want to know, have you ever seen the rain

Comin' down on a sunny day? Yeah

I want to know, have you ever seen the rain?

I want to know, have you ever seen the rain

Comin' down on a sunny day?

Fuente: Have you ever seen the rain - Creedence Clearwater Revival letra en inglés y español

El lenguaje no es solo palabras y gramática, sino también una cierta pronunciación, ritmo y emociones que no se pueden aprender de los libros; Desafortunadamente, el ritmo y las emociones del lenguaje no se reconocen en los libros. Las canciones siempre están llenas de ritmo y emociones del lenguaje del intérprete, lo que ayuda a estudiar el lenguaje al nivel de la memoria asociativa. El alumno no puede entender una sola palabra, pero siente las emociones que se transmiten a través de la canción y, a través de ellas, incluso adivina el significado del texto. Por lo tanto, enseñar una lengua extranjera con el uso de 
acompañamiento musical y metodológico es una tecnología psicológica y pedagógica importante y relevante.

Sobre las ventajas de introducir el acompañamiento musical y metodológico en el proceso de enseñanza del inglés en clases grupales de estudiantes. Al principio, cuando los estudiantes recién comienzan a aprender un idioma extranjero, las canciones en este idioma les parecen un conjunto simple de sonidos apenas distinguibles. Pero después de un tiempo escuchando música, los estudiantes comienzan a captar combinaciones similares de sonidos, pueden aislar palabras individuales sin siquiera comprender su significado, por lo que la música se prepara para el estudio serio de un idioma extranjero. Dado que a menudo las palabras y especialmente el coro en la canción se repiten muchas veces, la pronunciación, el vocabulario y la letra completa se recuerdan con bastante rapidez. Por lo tanto, los estudiantes sin estrés psicológico reponen su vocabulario y memorizan muchas frases y expresiones nuevas sin abarrotar, y además, aprenden a reconocer el habla inglesa de oído, que es una de las habilidades más necesarias e importantes para aprender un idioma extranjero.

\section{Conclusiones:}

- Durante el acompañamiento musical y metodológico de las clases de inglés, el maestro logra ampliar significativamente el vocabulario de los estudiantes.

- Los estudiantes aprenden nuevas expresiones interesantes que ayudarán a que su discurso oral sea más brillante y cercano al discurso de los hablantes nativos.

- El acompañamiento musical y metodológico le permite expresar emociones, trabajar en la melodía del texto y la entonación de diferentes frases.

- El método disminuye la sobrecarga emocional y el estrés de los estudiantes para adquirir información de una nueva lengua extranjera

- Las clases de acompañamiento musical en el idioma inglés le permiten al maestro y a los estudiantes ahorrar recursos de tiempo de estudio, memorizar vocabulario sin estrés.

\section{Referencias bibliográficas:}

Anaya-Durand, A. \&. -H. (2010). ¿Motivar para aprobar o para aprender? Estrategias de motivación del aprendizaje para los estudiantes. Tecnología, ciencia, educación, 25(1), 5-14.

Blasco, S. P. (2001). Importancia de la musicoterapia en el área emocional del ser humano. Revista interuniversitaria de formación del profesorado. (42), 91-113., (42), 91-113. 
Dandrea, F. D. (2018). Articulación Lingüística y Cultural Mercosur. Red de Integración Regional para ELSE en el contexto Mercosur, 45.

Espinoza, A. Q. (2015). El uso de canciones para el aprendizaje de la gramática en inglés como lengua extranjera. Horizontes Pedagógicos, 17(2), 8-16.

Inca, E. A. (2020). Análisis performativo de la andragogía lingüística del Inglés para el desarrollo socio-cultural. ConcienciaDigital, 3(2.1), 177-187.

Madariaga, J. M. (2011). Análisis de la práctica educativa del profesorado de música y su relación con la motivación del alumnado. Cultura y Educación, 23(3), 463-476.

Toscano Fuentes, C. M. (2012). La música como herramienta facilitadora del aprendizaje del inglés como lengua extranjera. 


\section{PARA CITAR EL ARTÍCULO INDEXADO.}

Inca Chunata, A. G., Colcha Guashpa, E. I., Guijarro Paguay, S. L., \& Martínez Paredes, L. M. (2020). Apoyo metodológico musical de las lecciones de inglés como tecnología pedagógica . Explorador Digital, 4(3), 6-17.

https://doi.org/10.33262/exploradordigital.v4i3.1275

\section{Ciencia \\ LDigital}

El artículo que se publica es de exclusiva responsabilidad de los autores y no necesariamente reflejan el pensamiento de la Revista Explorador Digital.

El artículo queda en propiedad de la revista y, por tanto, su publicación parcial y/o total en otro medio tiene que ser autorizado por el director de la Revista Explorador Digital.
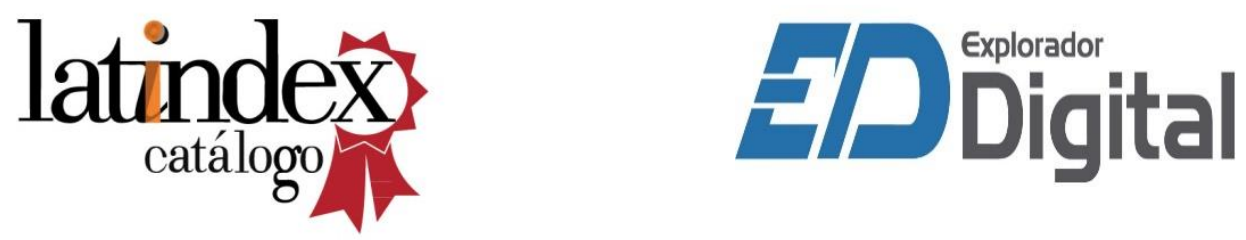